\title{
Joint Admission Control for Cooperative Cognitive Radio Networks
}

\author{
Jin Lai ${ }^{\dagger}$, Eryk Dutkiewicz ${ }^{\dagger}$, Ren Ping Liu ${ }^{\ddagger}$, Rein Vesilo ${ }^{\dagger}$ \\ ${ }^{\dagger}$ Department of Electronic Engineering, Macquarie University, Sydney, Australia \\ ${ }^{\ddagger}$ ICT Centre, Commonwealth Scientific and Industrial Research Organization (CSIRO), Sydney, Australia
}

\begin{abstract}
This paper studies the performance of admission control in cognitive radio networks (CRNs). We propose a CRN architecture featuring cooperation among several CRNs in the same geographical area. Joint Admission Control (JAC) enables secondary users (SUs) to have access to the combined spectrum pool of the cooperating CRNs. Three joint admission control schemes are investigated and quantitatively analyzed using continuous-time Markov chain analysis. Analytical results reveal new insights that the channel-aware admission control scheme achieves the lowest blocking probability at the expense of communication overhead for obtaining channel usage information in each CRN while the weighted selection scheme obtains the lowest forced termination probability. Moreover, we quantify the gain of cooperation through performance comparison between Joint Admission Control and separate admission control where SUs are restricted to using only one specific CRN. We demonstrate that JAC can achieve significant performance improvement.
\end{abstract}

\section{INTRODUCTION}

Cognitive radio $(\mathrm{CR})$ is envisaged as a novel technology to greatly improve spectrum unitization by enabling opportunistic access to the licensed spectrum band by secondary users (SUs). In cognitive radio networks (CRNs), primary users (PUs) take precedence over SUs to utilize the licensed spectrum band. SUs are only allowed to opportunistically operate in the licensed spectrum band whenever it is left unused by PUs. Prior to accessing a specific frequency band, SUs or their proxies have to sense the available spectrum. The SU has to vacate the occupied channel immediately whenever a PU transmission on the same channel is detected. The interrupted SU may switch to an unused spectrum band if possible or be forced to terminate if no free channel can be found [1]. This paper considers infrastructure-based primary and cognitive radio networks and investigates connection admission control in such networks.

A number of studies show the importance of admission control for SUs. In [6] a class-based call admission control model was proposed to reduce blocking probability for voice calls by giving them higher priority over data calls. In [7] optimal admission and eviction controls of $\mathrm{CR}$ users were proposed to maximize the profit for a secondary wireless service provider under the constraints of blocking and dropping probabilities. [8] indicated that without adequate admission control for SUs, sufficient service protection for PUs could not be guaranteed due to the occurrence of uncontrolled collisions. A collision-constrained network selection method was proposed to maximize system throughput subject to a given PU collision probability. However, PUs collision probability is unable to accurately reflect the performance of SUs. In [9] three spectrum admission control policies in dynamic spectrum sharing among different radio systems are proposed to increase the total supported traffic and improve spectrum utilization, However, this approach does not apply to opportunistic spectrum access scenarios. In [10][11] joint admission control and power control issues for CRNs are studied to maximize network revenue with guaranteed interference requirements.

Alternative methods for improving network performance for SUs include the following. In [2] Zhang proposed to utilize a buffering mechanism for CR subscribers to reduce blocking probability with very minor increased forced termination probability while in [3][4] channel reservation schemes are presented to reduce SU forced termination probability at the expense of a slight increase of blocking probability. In [5] a dynamic spectrum assignment scheme was proposed to reduce SU dropping probability and yield higher throughput via increasing the maximum allowable service rate. Previous research mainly considers scenarios where SUs have access to only a single CRN. Our study shows that in a single CRN, SUs are vulnerable to primary network traffic variations, which could lead to serious service degradation, particularly when relatively heavy traffic intensity occurs in the corresponding primary network. It is expected that in the future several CRNs with non-overlapping spectrum pools owned by different network operators may coexist in the same geographical area.

In this paper we propose a new CRN architecture that takes advantage of cooperation among such networks. A Joint Admission Control (JAC) model is presented for the optimum control of the overall network and spectrum access. We investigate and theoretically analyze three joint admission control schemes by developing continuous-time Markov chain analysis for the cooperative CRN model. The Markov chains are solved numerically, and our results reveal new insights on the performances of the admission control schemes. Our analytical results, validated through extensive simulations, show that the channel-aware selection scheme outperforms the random and weighted selection schemes at the expense of communication overhead for obtaining channel usage information in each CRN. Moreover, we quantitatively analyze the gain of cooperation between JAC and separate admission control (SAC) where SUs have access only to a single CRN.

The remainder of this paper is organized as follows. In Section II the performance issues of separate admission control are discussed. A Joint Admission Control model with three 
admission control schemes is subsequently proposed and theoretically analyzed in Section III. Numerical and simulation results are presented in Section IV. Finally Section V concludes this paper.

\section{ISSUE OF SEPARATE ADMISSION CONTROL}

In the separate admission control scenario SUs have access to only one CRN. In this model each channel in the spectrum pool is assumed to be identical in terms of capacity and other characteristics and spectrum handovers in the same CRN are supported. Each PU or SU can access only one channel at a given time. It is also assumed that PU (SU) arrivals follow a Poisson process while the channel holding times of PUs (SUs) are exponentially distributed. We investigate performance of separate admission control using Markov chain analysis and simulation. The details of the analysis is omitted due to space limitations.

In our investigation two identical channels are assumed in the CRN and the SU traffic intensity is set to 0.1 . The numerical results obtained by solving for the steady state probability of the Markov chain and the corresponding simulation results are presented in Fig. 1. As can be seen from the figure, the SU blocking probability and forced termination probability increase significantly while the SU completion probability declines dramatically when PUs traffic intensity increases from 0.1 to 1 . It can be seen that SUs might suffer from unacceptably low completion probability and high blocking probability as well as high forced termination probability which may result in great dissatisfaction for those users. This observation motivates our proposal for joint admission control by cooperating CRNs.

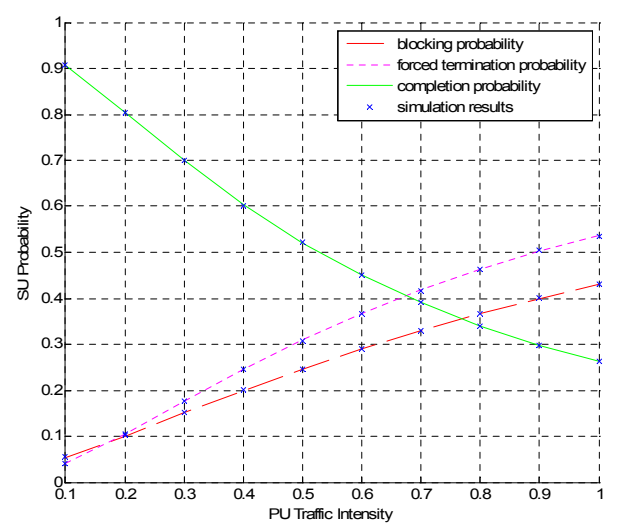

Fig. 1. Performance under Separate Admission Control

\section{JOINT ADMISSION CONTROL}

\section{A. Joint Admission Control Model}

A Joint Admission Control (JAC) model is proposed to improve SU performance by allowing cooperation among CRNs while both primary networks and primary users remain unaffected as shown in Fig. 2. Each CRN can be owned by a different network operator. Compared to the SAC model described in Section II where SUs can only access free channels in a single CRN, SUs in JAC may utilize any free channel in all the CRNs in the same geographical area. SU connection handovers between different CRNs are supported in the system.

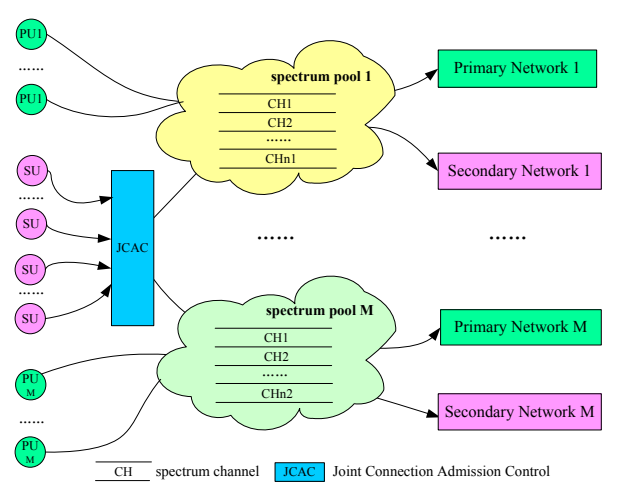

Fig. 2. Co-operative CRNs model with Joint Admission Control

The new entity where different admission control policies can be implemented is introduced to make an admission decision upon a SU arrival, shown as JCAC (Joint Connection Admission Control) in Fig. 2. JACA which can comminute with admission control entity of each CRN plays as central allocator for joint admission control functionality. The joint admission control can be carried out by multiple operators or provided as a service to $\mathrm{CR}$ users for network selection by a third party provider. Spectrum sensing before spectrum access by both base station and SU should be performed mandatorily. An incoming SU will be assigned to a CRN according to a predefined selection policy in JCAC. If the selected CRN does not have a free channel to accommodate this SU, the connection will be blocked. While a SU is holding a channel and a new PU requests to access this particular channel, the $\mathrm{SU}$ has to vacate the channel. If no free channel is available for the SU in the same CRN, the SU will attempt to switch over to a free channel in another CRN to continue the ongoing connection. If no free channel is available, the interrupted SU will be terminated. The completion of a SU connection will release the channel.

We assume that $C_{m}$ channels are available in CRN $m$. The arrival of PUs in CRN $m$ follows a Poisson process with mean arrival rate $\lambda_{p u \_} m$ while the PUs service time is exponentially distributed with mean $1 / \mu_{p u_{-} m}$. Similarly, SUs arrival rate follows a Poisson process with mean arrival rates $\lambda_{s u}$ and the SUs service time follows exponential distribution with mean $1 / \mu_{p u}$. It is also assumed that CRNs and SUs can perform perfect spectrum sensing and there exists a control channel between a CRN and a SU to facilitate channel assignment and channel switching between CRNs.

\section{B. Joint Admission Control Schemes}

In the following three joint admission control schemes, are considered: random, weighted, and channel-aware selection.

1) Random Selection: In this scheme, an incoming SU will be randomly assigned to a CRN no matter if the network 
has a free channel or not. This scheme does not consider the unbalance of traffic load in different CRNs and could result in serious service degradation of SUs when sharing the spectrum with PUs with heavy traffic intensity. However, this approach can be easily implemented since no prior knowledge of the network's condition is required.

2) Weighted Selection: In this approach, a weight $\omega_{m}$ is assigned to each CRN $m$ according to a predefined policy. The probability of selecting CRN $m$ is given by:

$$
P_{s, m}=\frac{\omega_{m}}{\sum_{1 \leq m \leq M} \omega_{m}}
$$

It is noted that heavy PU traffic intensity is bound to result in poor performance of the corresponding SUs. The heavier the primary network traffic intensity, the poorer the performance SUs will experience. Hence a reasonable approach to assign weights for different CRNs is to make the weights proportional to the available capacities.

3) Channel-aware Selection: In this scheme, upon a SU connection request, JCAC firstly determines the channel availability of each CRN. If several CRNs have at least one free channel, then the SU will be randomly allocated to one of them. This scheme requires JCAC to be able to obtain the knowledge of channel availability in each CRN which introduces communication overhead between JCAC and CRNs. Nonetheless, the benefit of this scheme is to achieve better system performance which is discussed in Section IV.

\section{Markov Model Analysis}

For simplicity we consider the network scenario consisting of two CRNs, however, our analysis can be readily extended to scenarios with more CRNs. Due to the limited space we only present the analytical model under the channel-aware selection scheme by developing a continuous time Markov chain. Suppose that $(i, j)$ represents the state of CRN 1, where $i$ and $j$ denote the number of channels occupied by PUs and SUs respectively at any time. Likewise $(m, n)$ is used to describe the state of CRN 2. Therefore, the joint state of the overall system can be referred to as $((i, j)(m, n))$ and the state space $\Gamma_{J A C}$ is defined by:

$$
\begin{gathered}
\Gamma_{J A C}=\left\{((i, j)(m, n)) \mid 0 \leq i \leq C_{n 1} ; 0 \leq j \leq C_{n 1}\right. \\
0 \leq i+j \leq C_{n 1} ; 0 \leq n \leq C_{n 2} \\
\left.0 \leq m \leq C_{n 2} ; 0 \leq n+m \leq C_{n 2}\right\}
\end{gathered}
$$

It is observed that the transitions among these finite states are triggered by the events of PU1/PU2 arrival and departure as well as SU arrival and departure. Suppose that state $((i, j)(m, n))$ is the current state, the resulting event analysis for PU1/SU arrival and departure is shown in Fig. 3.

1) PU1 arrival and departure: A PU1 connection will be accepted if one or more channels are unused in CRN 1. If all channels in CRN 1 are occupied by PUs and SUs, a new arriving PU1 will cause one of the SUs in CRN 1 to be evicted from its channel. Depending on channel availability in CRN 2, this interrupted SU might handover to CRN 2 to continue the ongoing connection or be dropped because of no free channel
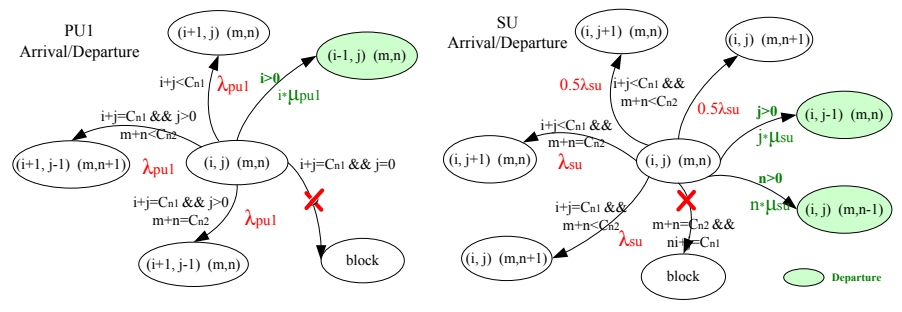

Fig. 3. Events analysis: PU1/SU arrival and departure

to switch to. These cases correspond to transitions into state $((i+1, j-1)(m, n+1))$ or $((i+1, j-1)(m, n))$. If all channels in CRN 1 are used by PUs, an incoming PU1 will be blocked. Upon a PU1 service completion, the occupied channel will be released in CRN 1.

2) PU2 arrival and departure: A PU2 arrival and departure are similar to a PU1 arrival and departure except they take place in CRN 2 instead of CRN 1.

3) SU arrival and departure: For a SU arrival, if both CRNs have one or more free channels, an incoming SU will be accepted with the same probability of selecting CRN 1 or CRN 2 , transiting into state $((i, j+1)(m, n))$ or $((i, j)(m, n+1))$, respectively. On the other hand, if only one CRN has a free channel, a new arriving SU has no choice but to enter the CRN with the free channel. In addition, if the total number of channels used by either PUs or SUs equals $C_{n 1}+C_{n 2}$, a SU connection request will be blocked as a result of no available channels. Upon a SU service completion, the used channel will be released in the corresponding CRN.

4) State transition diagram: We illustrate one case where the state satisfies $i=C_{n 1} \& \& m+n<C_{n 2} \& \& \mathrm{~m}>0 \& \& \mathrm{n}>$ 0 , shown in Fig. 4, as an example to demonstrate the state transition diagram. Note that the transition from $((i-1, j+$ $1)(m, n-1))$ into $((i, j)(m, n))$ means that a SU in CRN 1 will be handed over to CRN 2. We define $\pi((i, j)(m, n))$ as the steady-state probability for each state $((i, j)(m, n)) \in \Gamma_{J A C}$. Therefore, the balance equation can be given by:

$$
\begin{aligned}
& \left(\lambda_{p u 2}+\lambda_{s u}+C_{n 1} \mu_{p u 1}+m \mu_{p u 2}+n \mu_{s u}\right) \pi_{\left(\left(C_{n 1}, 0\right)(m, n)\right)} \\
& =(m+1) \mu_{p u 2} \pi_{\left(\left(C_{n 1}, 0\right)(m+1, n)\right)}+\lambda_{p u 2} \pi_{\left(\left(C_{n 1}, 0\right)(m-1, n)\right)} \\
& +\lambda_{s u} \pi_{\left(\left(C_{n 1}, 0\right)(m, n-1)\right)}+(n+1) \mu_{s u} \pi_{\left(\left(C_{n 1}, 0\right)(m, n+1)\right)} \\
& +\lambda_{p u 1} \pi_{\left(\left(C_{n 1}-1,0\right)(m, n)\right)}+\lambda_{p u 1} \pi_{\left(\left(C_{n 1}-1,1\right)(m, n-1)\right)}
\end{aligned}
$$

By analyzing all the events and state transition diagrams, transition rate matrix $Q_{J A C}$ can be obtained. We then have [13]:

$$
\prod Q_{J A C}=0
$$

where $\prod=\left[\pi_{((0,0)(0,0))}, \ldots, \pi_{((i, j)(m, n))}, \ldots, \pi_{\left(\left(C_{n 1}, 0\right)\left(C_{n 2}, 0\right)\right)}\right]$ is the steady-state probability vector. In addition, the summation of all steady-state probabilities satisfies the normalization constraint $\sum_{((i, j)(m, n)) \in \Gamma_{J A C}} \pi_{((i, j)(m, n))}=1$. Therefore, the steady-state probability vector can be obtained by solving the system of the corresponding linear equations. For this paper we numerically solve the above Markov chain by using the least-square QR factorization algorithm and derive the steadystate probability vector. 


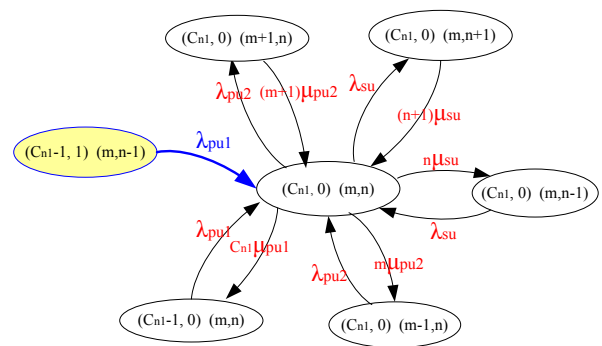

Fig. 4. Events analysis: PU1/SU arrival and departure

\section{Performance Metrics}

1) SU blocking probability: A SU will be blocked when all channels in both CRNs are occupied by either PUs or SUs. Therefore, SU blocking probability is given by:

$$
P_{b l k}=\sum_{i \geq 0, j \geq 0, i+j=C_{n 1}, m \geq 0, n \geq 0, m+n=C_{n 2}} \pi_{((i, j)(m, n))}
$$

2) SU forced termination probability: Once a SU connection request has been accepted, it can still be dropped during its service time as a result of a PU arrival and no free channel available for the SU. Hence SU forced termination probability [12] defined as the conditional probability of terminated SUs rate under accepted SUs rate is given by:

$$
\begin{aligned}
& P_{f t}=\left(\sum_{i \geq 0, j>0, i+j=C_{n 1}, m+n=C_{n 2}} \pi_{((i, j)(m, n))} \lambda_{p u 1}+\right. \\
& \left.\sum_{i+j=C_{n 1}, m \geq 0, n>0, m+n=C_{n 2}} \pi_{((i, j)(m, n))} \lambda_{p u 2}\right) /\left(\left(1-P_{b l k}\right) \lambda_{s u}\right)
\end{aligned}
$$

3) SU completion probability: A SU connection request will be either blocked or terminated or completed. Following the definition of non-completion probability in [2], SU completion probability is given by:

$$
P_{c m p l}=\left(1-P_{b l k}\right)\left(1-P_{f t}\right)
$$

\section{NUMERICAL AND SIMULATION RESULTS}

This section presents numerical and simulation results for the three JAC schemes. Performance comparisons between SAC and JAC are also presented.

\section{A. Performance Comparison Among Three JAC Schemes}

Without loss of generality, we assume that PU1 traffic intensity is heavier than PU2 traffic intensity with PU1 traffic intensity set to 0.9 and PU2 traffic intensity set to 0.1 . In the weighted selection scheme, the weights for the two CRNs are 0.1 and 0.9 , respectively. The SU traffic intensity is set to the range from 0.05 to 0.5 with increments of 0.05 . Two channels are assumed in each of the two CRNs.

As shown in Fig. 5-7, SU blocking probability and forced termination probability increase steadily while SU completion probability decreases significantly when the SU traffic intensity increases regardless of the JAC scheme used. However, for a given SU traffic intensity, the weighted selection scheme gets the lowest forced termination probability the channelaware selection scheme always achieves the lowest blocking probability and highest completion probability but it has a

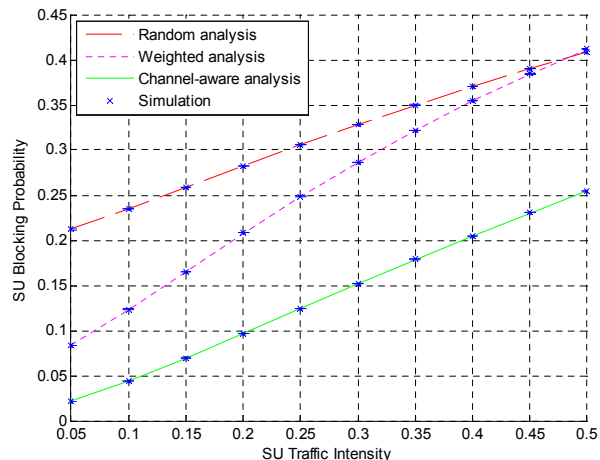

Fig. 5. Blocking probability for three JAC schemes

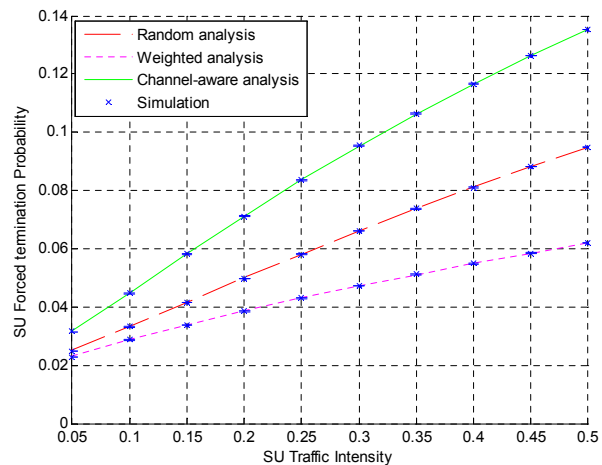

Fig. 6. Forced termination probability for three JAC schemes

slightly higher chance of being terminated. However, such increase in forced termination probability is compensated by the reduction in SU blocking probability.

Comparison between the analytical and simulation results (with 95\% confidence interval level) in Fig. 5-7 illustrates an excellent agreement.

\section{B. Performance Comparison Between SAC and JAC}

From the overall system perspective, for a given SU traffic intensity, we investigate the performance comparison between SAC and JAC while varying PU1 and PU2 traffic intensity. In $\mathrm{SAC}, \mathrm{SU}$ traffic is equally split to each CRN, while in JAC SU traffic is assigned to a CRN according to the channel-aware scheme. SU traffic intensity for the entire system is set to 0.1 . Ten channels are assumed in each of the two CRNs.

According to Fig. 8 the SU blocking probability surface of JAC is much lower than that of SAC because JAC always selects the CRN with free channels. As can be seen from Fig. 9, the SU forced termination probability surface of JAC is also much lower than that of SAC since the interrupted SU may utilize free channels in the other CRN. Compared to the SAC scheme, the SU completion probability under JAC, as shown in Fig. 10, is significantly improved. For example, when traffic intensity of PU1 and PU2 is 0.8 and 0.4 respectively, SU completion probability can be improved by $37 \%$. High SU completion probability indicates that network operators can significantly increase their revenue while also improving users' experience. 


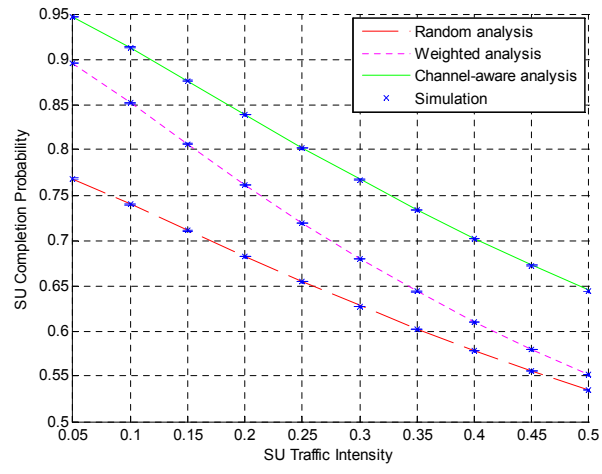

Fig. 7. Completion probability for three JAC schemes

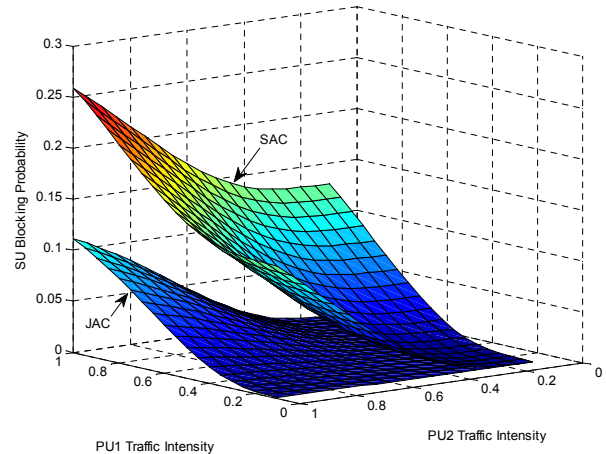

Fig. 8. SU blocking probability under SAC and JAC

\section{CONCLUSION}

In this paper we propose a new Joint Admission Control (JAC) model for cognitive radio networks (CRNs) that takes advantage of cooperation among CRNs to improve overall system performance. We examine three joint admission control schemes: random, weighted and channel-aware, through Markov chain analysis. The analytical results, validated through extensive simulations, show that the channelaware selection scheme outperforms the other schemes in terms of SU completion probability at the expense of some communication overhead to obtain CRNs network status. Performance comparison between JAC and separate admission control shows that significant performance improvements can be achieved through cooperation between cognitive radio network operators.

The proposed JAC model can be implemented as CRN cooperation entity by a third party operator to provide SU access to multiple CRNs. Future work will focus on expanding the model to multi-service scenarios with time-varying bandwidth connection requirement. Performance of the system will also be analyzed taking into account the signaling and handover costs associated with CRNs cooperation.

\section{REFERENCES}

[1] I. F. Akyildiz, W. Y. Lee, M. C. Vuran and S. Mohanty, "NeXt generation/ dynamic spectrum access/cognitive radio wireless networks: A survey", Computer Networks, vol.50, 2006.

[2] Y. Zhang, "Dynamic Spectrum Access in Cognitive Radio Wireless Networks", in Proc. IEEE ICC, May 2008.

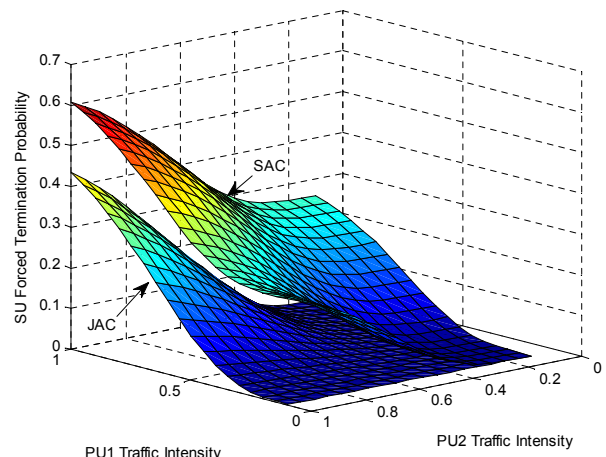

Fig. 9. SU forced termination probability under SAC and JAC

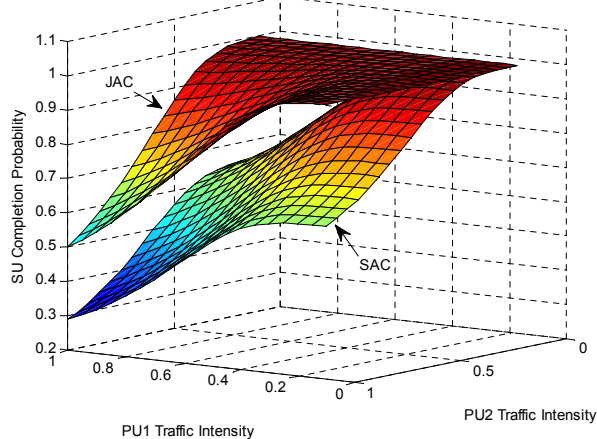

Fig. 10. SU completion probability under SAC and JAC

[3] X. Zhu, L. Shen and T.-S. P. Yum, "Analysis of Cognitive Radio Spectrum Access with Optimal Channel Reservation", IEEE Communication Letters, vol.11, no.4, April 2007

[4] D. Pacheco-Paramo, V. Pla, and J. Martinez-Bauset, "Optimal Admission Control in Cognitive Radio Networks", in Proc. IEEE CROWNCOM, June 2009.

[5] S. M. Kannappa and M. Saquib, "Performance Analysis of a Cognitive Network with Dynamic Spectrum Assignment to Secondary Users", in Proc. IEEE ICC, May 2010.

[6] D. Xue, H. Yu, X. Wang, and H. H. Chen, "Adoption of Cognitive Radio Scheme to Class-based Call Admission Control", in Proc. IEEE ICC, June 2009.

[7] H. Kim and K. G. Shin, "Optimal Admission and Eviction Control of Secondary Users at Cognitive Radio Hotspots", in Proc. IEEE SECON, June 2009

[8] C. Wang, K. Sohraby, R. Jana, L. Ji, and M. Daneshmand, "Network selection in cognitive radio systems", in Proc. IEEE GLOBECOM, December 2009

[9] P. K. Tang, Y. H. Chew, W. L. Yeow, and L. C. Ong, "Performance Comparison of Three Spectrum Admission Control Policies in Coordinated Dynamic Spectrum Sharing Systems", IEEE Transaction on Vehicular Technology, vol.58, No.7, September 2009.

[10] J. Xiang, Y. Zhang, and T. Skeie, "Joint admission and power control for cognitive radio cellular networks", in Proc. International Conference on Communication Systems (ICCS), 2008

[11] L. Zhang, Y. C. Liang and Y. Xin, "Joint Admission Control and Power Allocation for Cognitive Radio Networks", in Proc. IEEE International Conference on Acoustics, Speech and Signal Processing (ICASSP), 2007

[12] W. Ahmed, J. Gao, H. A. Suraweera and M. Faulkner, "Comments on 'Analysis of Cognitive Radio Spectrum Access with Optimal Channel Reservation",, IEEE Transaction on Wireless Communication, vol.8, No.9, September 2009.

[13] L. Kleinrock, "Queueing System volume I: Theory", by John Wiley \& Sons, 1975. 